\title{
Application of robo-pigeon in ethological studies of bird flocks
}

\author{
Hao Wang ${ }^{1,2, *}$, Jin $\mathrm{Wu}^{1}$, Ke Fang ${ }^{1}$, Lei Cai ${ }^{2, *}$, Lai-Shan Wang ${ }^{1}$ and Zhen-Dong Dai ${ }^{1}$ \\ ${ }^{1}$ Jiangsu Provincial Key Laboratory of Bionic Functional Materials, College of Mechanical and Electrical Engineering, \\ Nanjing University of Aeronautics and Astronautics, Nanjing, 210016, P. R. China \\ ${ }^{2}$ Shandong Provincial Key Laboratory of Biosensors, Biology Institute, Qilu University of Technology (Shandong Academy of
} Sciences), Jinan, 250103, P. R. China

*Correspondence: haowang@nuaa.edu.cn (Hao Wang); cailei@sdas.org (Lei Cai)

DOI:10.31083/i.jin.2020.03.159

This is an open access article under the CC BY 4.0 license (https://creativecommons.org/licenses/by/4.0/).

Birds flying collectively is a fascinating phenomenon in nature, which is central in ethological studies. Owing to the difficulty of introducing controlled variables into a natural bird flock, current animal behavior paradigms limit our understanding of collective behavior and mechanism. The recently developed technology of robo-pigeon, which allows behavior regulation over organisms through brain microstimulation, can potentially serve to design the controlled variables. However, it still poses challenges for unrestrained animals outdoors. Here we report the first application of robo-pigeon to the study of collective behavior, illustrating how intact pigeons in a flock interact with a program-controlled robo-pigeon. The controlled variable of direction manipulation introduced by the robo-pigeon may balance their preferred directional choice in the flock. Its effectivity depends on the hierarchical level to which the robo-pigeon belongs. This study suggests that direct manipulation of flight trajectories by a robo-pigeon might be a useful causal tool to study the collective behavior of bird flocks.

\section{Keywords}

Robo-pigeon; robo-animal; collective behavior; brain microstimulation; bird flocks; ethology; brain-computer interface

\section{Introduction}

Collectively moving animals, such as the African wildebeests under mass migration, the Pacific sardines under collective defense, and the country starlings under aerial performance, exhibit their fascinating collaborative behavior, broadly crossing the species (Vicsek and Zafeiris, 2012). Collectively flying birds, either inline formations or in cluster formations (Heppner, 1974), have inspired both engineers to reveal the aerodynamic efficiency under wake interactions (Bajec and Heppner, 2009; Dimock and Selig, 2003; May, 1979; Nathan and Barbosa, 2008; Weimerskirch et al., 2001), and biologists to understand the social cooperation among the individuals (Attanasi et al., 2014; Chen et al., 2017; Codling et al., 2007; Li et al., 2016; Nagy et al., 2010; Wilson and
Wilson, 2007; Zhang et al., 2014). Many experimental techniques to understand animals collective behavior rely on natural observation (Weitz et al., 2012).

However, there are several approaches that are capable of introducing a control variable through an agent. One is to construct a mechanical agent such as a bionic robot, albeit mobility limits its application. The other is to construct a digital or virtual agent relying on the technique of virtual reality and its application in animal behavior studies (Fry et al., 2008; Schultheiss et al., 2017; Stowers et al., 2017). In a zebrafish swarm study, for example, a digital agent was designed photo-realistically to mimic zebrafish, by which the directional choice mechanism with social interaction was discovered (Stowers et al., 2017). Recent investigations on robo-animals, crossing broad species (Aravanis et al., 2007; Cai et al., 2015; Erickson et al., 2015; Kobayashi et al., 2009; Sato and Maharbiz, 2010; Seo et al., 2017; Su et al., 2012; Talwar et al., 2002; Wang et al., 2018; Yang et al., 2017; Zhao et al., 2019), have paved another possible way to construct a hybrid agent, that is a whole organism with a controllable neuromuscular system, through brain microstimulation using custom-designed codes.

Robo-pigeon, as the flyable representative of robo-animals, has been studied by two distinct approaches. First, (Su et al., 2012; Yang et al., 2017) is imposing microsimulation into nerve nuclei or regions along the ascending pathway and feeling center, such as the dorsalis intermedius ventralis anterior (DIVA) with somatosensory function (Schneider and Necker, 1996), the periaqueductal gray (PAG) for pain or threaten feeling (Kingsbury et al., 2011). Second, (Cai et al., 2015; Seo et al., 2017; Wang et al., 2018; Zhao et al., 2019) is imposing microsimulation into nerve nuclei or regions along descending pathway, such as the formatio reticularis medialis mesencephali (FRM) that is located in the midbrain and related mainly to limbic functions (Medina and Reiner, 1997). Both approaches have been validated by field testing (Wang et al., 2018; Yang et al., 2017). The flight trajectories of robo-pigeons were modulated by altering turning directions using the command of the brain microstimulation. The latter approach was even able to elicit orbiting flight (Wang et al., 2018) by delivering a sequence of microsimulation to FRM. 
In this paper, with the technique of robo-pigeon (Wang et al., 2018), we first introduced the controlled variables of direct manipulation into a flying pigeon flock in a natural open space. We tested how the pigeon flock interacts with the program-controlled robo-pigeon. The controlled variables interferes with the preferred directional choice of the pigeon flock, and this interference depended on the hierarchical level to which the robo-pigeon belongs. This study demonstrates that the technology of robo-pigeon is a robust causal tool with broad application potentiality in collective behavior studies.

\section{Materials and methods}

\subsection{Animals and experimental protocols}

Homing pigeons (Columba livia) were cultured in a loft under a typical day/night light cycle. All of them were trained daily flying around the loft twice a day, equipped with dummy weight ( $16 \mathrm{~g}$, nearly the same size and weight as the onboard control module), to habituate them to fly and live with a load. The load of the dummy weight or the onboard control module was typically attached to the pigeon's back by gluing Velcro straps to the body of the pigeon and then attaching the load to the Velcro. Twenty pigeons, all between 2 and 3 years old, were chosen for long-distance homing training twice per week so that they all had previous homing experience before testing. The homing flight spanned over 10 kilometers between Jiangjun- and Zhongshan-Mountain in Nanjing, of which the terrain is mostly gently undulating or somewhat flat. Pigeons were taken to a release site near Jiangjun-Mountain in a van in carrier baskets allowing adequate ventilation. All tests were conducted on sunny days with winds less than third class. Since those pigeons did not always fly with a stable flock, only those animals $(\mathrm{N}=6)$ that constituted a stable group were chosen for this collective behavior study. Two individuals at the distinctly different hierarchical levels, i.e., high and low, were candidates for being robo-pigeons.

All experimental animals operating procedures are in line with the Guide of Laboratory Animal Management Ordinance of China and are approved by the Jiangsu Association for Laboratory Animal Science (Jiangsu, P. R. China).

\subsection{Hierarchical level of each bird in a flock}

To determine the hierarchical level of each individual in a flock, a custom-designed algorithm was developed (Matlab, The MathWorks, Inc., Natick, MA, USA) referring to the directional correlation method (Nagy et al., 2010). We first calculated the directional correlation delay for each pair of pigeons $i$ and $j(i \neq j)$, which formed a $6 \times 6$ upper triangular matrix of delay (see Results). The directional correlation delay for a pair is $C_{i j}(\tau)=$ $\left\langle\overrightarrow{v_{i}}(t) \bullet \overrightarrow{v_{j}}(t+\tau)\right\rangle$, where $\overrightarrow{v_{i}}(t)$ is the normalized velocity of bird i and $\bullet$ is the dot product. The maximum value of the $C_{i j}(\tau)$ correlation function, noted by $\tau_{i j}^{*}$, is the directional correlation delay time. When $\tau_{i j}^{*}$ is positive, it means the flight direction of the bird $\mathrm{j}$ falls behind that of the bird $\mathrm{i}$, which indicates that the hierarchical level of bird $\mathrm{i}$ is higher that of bird $\mathrm{j}$. All values of $\tau_{i j}^{*}$ constitutes a $6 \times 6$ matrix of delay. Since $C_{i j}(\tau)=C_{j i}(-\tau)$ and consequently $\tau_{i j}^{*}=-\tau_{j i}^{*}$, the matrix is indeed a symmetric matrix and can be represented as an upper triangular matrix with all positive values. The bird represented by the first row means its flight direction goes ahead all others of the flock, which indicates the highest hierarchical level of the bird.

\subsection{Robo-pigeons}

Two animals, at the highest and the lowest hierarchical level, were chosen for fabricating robo-pigeons, respectively. The surgical operation and electrode implantation in preparing the robopigeons was the same as our previous work (Cai et al., 2015; Wang et al., 2018). All efforts were made to minimize animal suffering and minimize the number of individuals used. For this study, only the FRM (Wang et al., 2018) was selected as the target of microstimulation. Whether the robo-pigeon works properly is assessed by the appearance of a complete circling flight trajectory during the brain microstimulation. The purpose of robo-pigeons is to introduce the controlled variables related to turning directly into a natural homing flight flock. For each trial of the flock flight, only one was enabled to receive the stimulation commands, while the other was deactivated just like an untreated bird.

\subsection{Temporal-spatial relations between a robo-pigeon and a rest of the flock}

To evaluate whether robo-pigeon influences the direction choice of a natural flying flock, besides its hierarchical level, we also calculate the temporal-spatial relations between the robopigeon and the rest of the flock, referring to the directional correlation method (Nagy et al., 2010). The temporal relation, denoted by advanced time, is the directional correlation delay for the robopigeon concerning the average direction of motion of the rest of the flock. The directional correlation function for a robo-pigeon $\mathrm{k}$ and a rest of the flock is $C_{k}(\tau)=\left\langle\overrightarrow{v_{k}}(t) \bullet \overrightarrow{v_{j}}(t+\tau)\right\rangle_{k, j}$, and the maximum value of this function is the advanced time $\tau_{k}^{*}$. The spatial relation, denoted by advanced distance, presents the relative spatial position of a robo-pigeon to the rest of the flock. We calculated for each robo-pigeon the average projected distance onto the direction of motion of the whole flock as $d_{k F}=\left\langle d_{k F}(t)\right\rangle_{t}$ and $d_{k F}(t)=\left(\overrightarrow{x_{k}}(t)-\overrightarrow{x_{F}}(t)\right) \bullet \overrightarrow{v_{a l l}}(t)$, where $\overrightarrow{x_{k}}(t)$ is the position of robo-pigeon $\mathrm{k}, \overrightarrow{x_{F}}(t)$ is the mean position of the rest of the flock, and $\overrightarrow{v_{a l l}}(t)$ is the normalised velocity of the whole flock.

\subsection{Control module and stimulation target}

The control module was updated from the one we used before (Wang et al., 2018) and was slightly improved. The total mass of the control module, including a rechargeable lithium battery, was reduced to $11.8 \mathrm{~g}$, and the mean size was reduced to $30 \mathrm{~mm} \times 30$ $\mathrm{mm} \times 12 \mathrm{~mm}$. Two functions were integrated into the module, flight trajectory recording, and brain microstimulation. The formation of reticularis medialis mesencephali (FRM) was used as the target of brain microstimulation (Wang et al., 2018).

\section{Results \\ 3.1 Hierarchical levels inside the pigeon flock}

The pigeon flock with six individuals (Table 1) flew several times in the test zone (Fig. 1), and their flight trajectories were recorded to identify the hierarchical level of each individual. Since the directional correlation delay time was indistinguishable during straight flight, only the flight sections with apparent maneuvers, such as circling or sharp turning, were taken to analyze the hierarchical relationships among the individuals. The inset (A) and (B) in Fig. 1 shows one example of the flight section of circling, and 


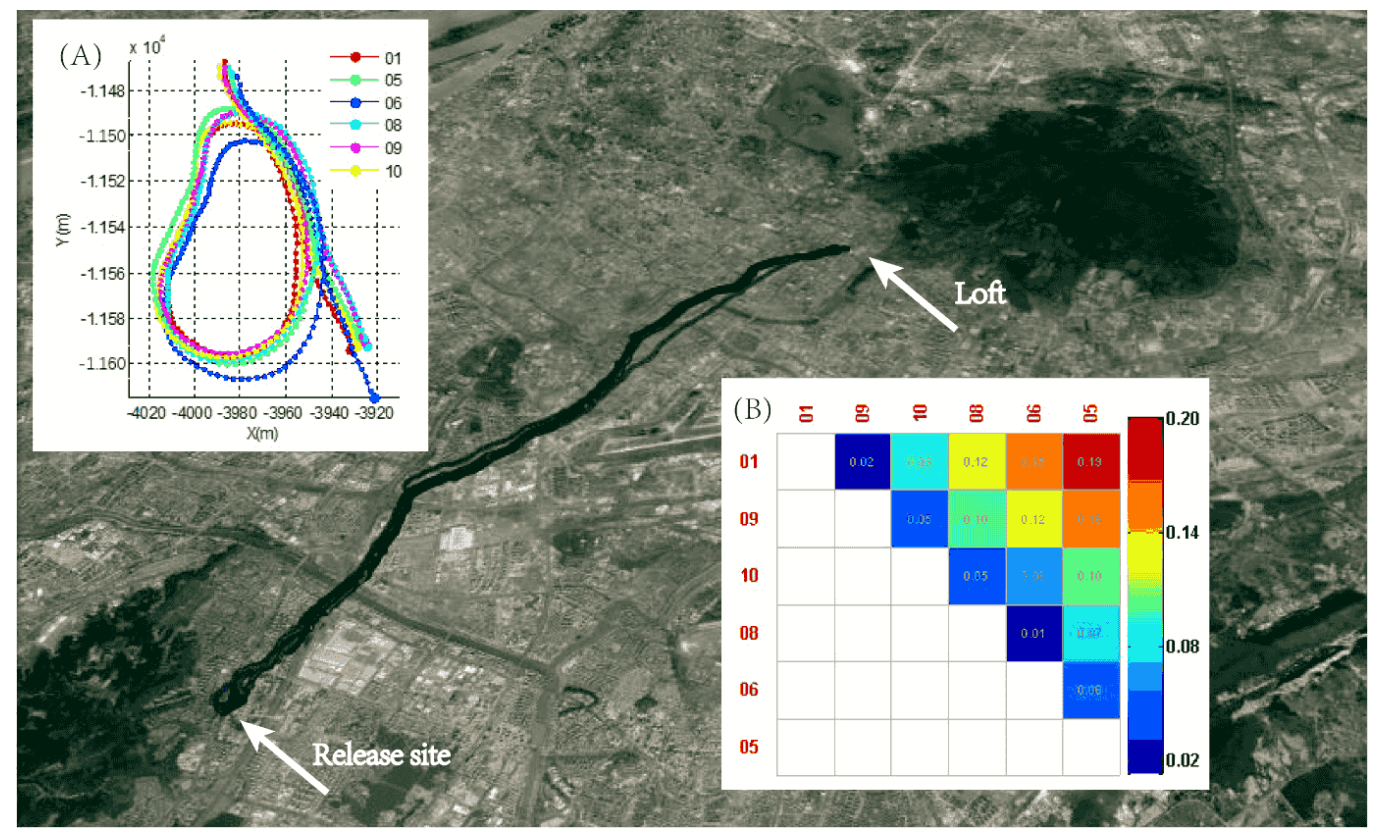

Fig. 1. A homing flight example of a flock of six pigeons. Different colors represent flight trajectories. The distance between the release site and the loft (denoted by white arrows) is over 10 kilometers. The inset (A) shows a 30-second segment from the free flight, illustrating a complete circling. The negative values in both $\mathrm{x}$ - and $\mathrm{y}$-axis indicate the birds were flying in the south-west of the loft set as the origin. The corresponding hierarchical level analysis is shown in the inset (B)-the upper triangular matrix of delay calculated by the directional correlation method. The number presented by the color index and shown in each cell indicates the delay (unit: s). Matlab generated the two insets, and the background was a screen-capture of Google Earth with the loaded flight trajectories.

Table 1. Mass and the hierarchical level of each individual in the pigeon flock

\begin{tabular}{lccccccc}
\hline Bird ID & \#01 & $\# 05$ & $\# 06$ & $\# 08$ & $\# 09$ & $\# 10$ \\
\hline Mass (g) & 468 & 501 & 408 & 469 & 452 & 456 \\
Mean hierarchical level & 1.9 & 3.3 & 3.9 & 4.7 & 3.2 & 4.0 \\
Possibility in the top 3 levels (\%) & 90 & 40 & 40 & 20 & 60 & 40 \\
Possibility in the last 3 levels (\%) & 10 & 60 & 60 & 80 & 40 & 60 \\
Possibility in the top 2 levels (\%) & 80 & 40 & 30 & 10 & 20 & 20 \\
Possibility in the last 2 levels (\%) & 10 & 30 & 50 & 60 & 10 & 40 \\
\hline
\end{tabular}

the corresponding upper triangular matrix of delay indicating the order of the hierarchical levels. The matrix shows that all others followed bird \#01 with $0.02 \mathrm{~s}, 0.09 \mathrm{~s}, 0.12 \mathrm{~s}, 0.15 \mathrm{~s}$, and $0.19 \mathrm{~s}$ delay, respectively, which implied the first hierarchical level of bird \#01 inside the flock. Bird \#09 followed bird \#01 but followed by the rest four birds, so it was at the second hierarchical level.

Similarly, the hierarchical level for all individuals was derived from this upper triangular matrix directly, and it was \#01, \#09, $\# 10$, \#08, \#06, and \#05 in descending order. Using a data pool with ten flight sections, we calculated the averaged hierarchical level (Table 1). The minimum value (1.9) indicated the bird (\#01) was the highest hierarchical level, while the maximum value (4.7) the lowest hierarchical level of bird \#08. Table 1 also gives the probability of occurrence of each individual in the front (the first 2 or 3 hierarchical levels) or rear (the last 2 or 3 hierarchical levels) of the flock. The bird \#01 appeared in the first 2 and 3 hierarchical levels in the possibility of $80 \%$ and $90 \%$, respectively; while the bird \#08 appeared in the last 2 and 3 hierarchical levels in the possibility of $60 \%$ and $80 \%$, respectively. These results show different hierarchical levels of bird \#01 and birds \#08 in the flock, which provides good attributes as candidates for robo-pigeons in the comparative study of the influences of manual controlled variables to the collective bird motion.

\subsection{Manually controlled robo-pigeons}

Bird \#01 and bird \#08, at high and low hierarchical levels, respectively, were modified to robo-pigeons surgically (Cai et al., 2015). Each robo-pigeon (see Fig. S1) was competent to carry a control module with a brain-computer interface. The control module ran preprogrammed computer codes that could trigger brain microstimulation commands when the flight condition met the predefined criteria. The commands consequently elicited certain maneuvers such as circling in the bird (Wang et al., 2018). In this way, the robo-pigeon introduced some motion-related controlled variables (circling here) into a freely flying flock, just like a manually controlled command.

Before collectively flying in field tests, we validated the controllability of each robo-pigeon while they flew alone. It was defined as a positive control if the robo-pigeon could make a complete circling within the duration of one sequence of microstimulation (Wang et al., 2018). Both of the two robo-pigeons performed prominently. For their first ten flights under microstimulation, the robo-pigeon \#01 was elicited ten circles (100\% success rate) and the robo-pigeon \#08 nine circles (90\% success rate), respectively.

\subsection{Flock flights under the influence of robo-pigeons}

The pigeon flock was composed of six individuals (\#01, \#05, $\# 06, \# 08, \# 09$ and \#10), among which pigeon \#01 and pigeon \#08 

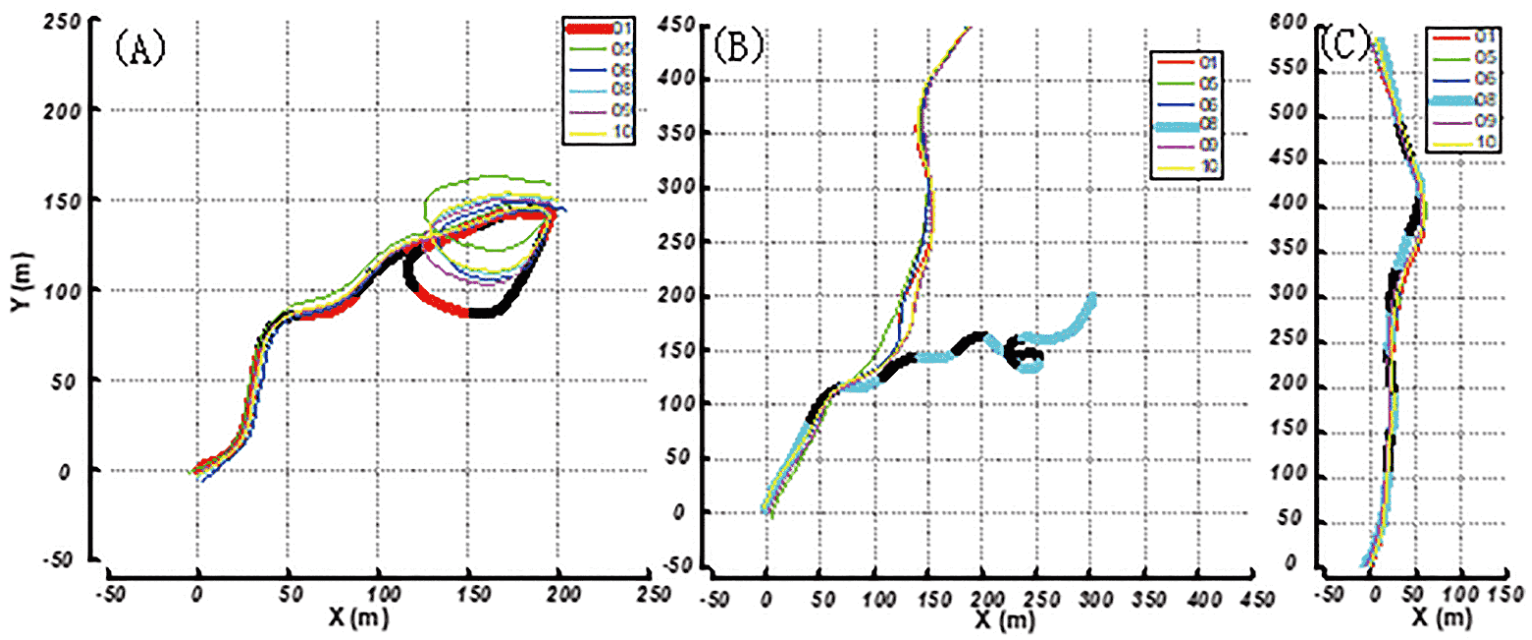

Fig. 2. Flight trajectories of the pigeon flock under the influence of robo-pigeons. The color curves represent animals' flight trajectories, and the thick one is the robo-pigeon with a running control module. The black parts one the curve indicates the time when the brain microstimulation was onset. (A) An example of successful flock circling induced by robo-pigeon \#01. (B) The robo-pigeon \#08 made circling but out of the rest flock. (C) Robo-pigeon \#08 followed the flight trajectory of the rest flock.

Table 2. The hierarchical relationship between robo-pigeon \#01 and the flock

\begin{tabular}{ccccc}
\hline Trial & Hierarchical order & Coefficient $\left(C_{k}\right)$ & Advanced distance $\left(d_{k F}\right.$ in m) & Advanced time $\left(\tau_{k}^{*}\right.$ in s $)$ \\
\hline 1 & $01-05-10-06-09-08$ & 0.96 & 0.8 & 7.68 \\
2 & $01-09-10-08-05-06$ & 0.77 & 1.4 & 0.20 \\
3 & $01-09-06-08-10-05$ & 0.98 & 0.1 & 1.29 \\
4 & $01-10-09-06-08-05$ & 0.53 & 0.4 & 1.03 \\
5 & $01-06-10-09-08-05$ & 0.96 & 0.5 & 0.57 \\
6 & $01-10-06-05-09-08$ & 0.48 & 0.2 & 11.03 \\
\hline
\end{tabular}

were robo-pigeons. Each of the two could be activated to introduce motion-related controlled variables into the flock, by means of running the control module it carried. One and only one activated robo-pigeon existed in the flock for each trial. Totally twenty trials were tested for the two cases, the flock with the activated robo-pigeon \#01 (see Fig. S2) or with the activated robo-pigeon \#08 (see Fig. S3), ten for each.

The robo-pigeon \#01, at the high hierarchical level, could easily affect the flock's decision of flight direction by its induced circling (Fig. 2A). The rest of the flock flew a circle following the robo-pigeon \#01. Comparatively, the robo-pigeon \#08, at the low hierarchical level, could hardly affect the flock overall. The robopigeon \#08 either flew (elicited by microsimulation) circling but was out of the rest flock (Fig. 2B), or even performed no circling and turned to follow the flight of the rest flock (Fig. 2C).

Whether introduced controlled variables through a robo-pigeon could affect the flock successfully was judged by the appearance of circling after the microstimulation over the robo-pigeon. The flock flying with the robo-pigeon \#01 achieved a $60 \%$ success rate, while those with the robo-pigeon \#08 showed no circling, i.e., $0 \%$ success rate (see Fig. S2 and S3). All the successful trials are listed in Table 2.

\section{Discussion}

\subsection{How a robo-pigeon affects the flight of the flock}

For all these successful trials (Table 2), robo-pigeon \#01 was always stay at the highest hierarchical level, which implied its strong affection over the flock. After the induced circling or the microstimulation was released, the robo-pigeon \#01 and the rest of the flock resumed natural flying towards the loft. In all cases except trail 6, the robo-pigeon \#01 remained at the top of the hierarchy. The directional correlation coefficient between the flight trajectory of the robo-pigeon and that of the rest of the flock varied a lot. It relied on the severity of the flight maneuver induced by the microstimulation in the robo-pigeon. When the induced circling and turning were finely smooth, such as in trial 3 (shown in Fig. 2a), the rest of the flock could follow the robo-pigeon timely, so the two curves of the flight trajectory could correlate reasonably. Nevertheless, when the modulated circling and turning were quite dramatic, such as in trial 4 (also see Fig. S2F), the rest of the flock had no enough time to respond to the robo-pigeon. This suggested that there might be up to limit of flight velocity and acceleration in the dynamic response in collective motions. The advanced distance and time were always positive though they varied a lot, which implies that the robo-pigeon got well ahead of the rest of the flock in position and time. This supports the high hierarchical level of robo-pigeon \#01 from a different view.

There was no successful circling induced by robo-pigeon \#08 (see Fig. S3), near the bottom of the flock hierarchy. Though, in 
some cases, such as Fig. S3F/Fig. 2B, Fig. S3G, and Fig. S3I, the flock briefly turned when robo-pigeon \#08 was controlled to turn at the very beginning. The duration was too short (no more than $2 \mathrm{~s}$ or 10 sample points) to affect the robo-pigeon's hierarchy level in the whole flight section. However, it suggested that even a low-hierarchy bird might have a mild, transient influence on the flock, which needs to be addressed by more delicate studies and maybe an interesting phenomenon in social animals.

This implies one of the feasible criteria for choosing a robopigeon to introduce controlled variables into a flock. Though any individual in a flock can be rebuilt into a robo-pigeon by the technique of the brain microstimulation, not all of them can introduce controlled variables into the flock as a hybrid agent. Only robopigeon at a high hierarchical level can introduce controlled variables into the flock, and consequently to affect its decision of direction choice.

\subsection{Another way to design a robo-pigeon}

Brain microstimulation is undoubtedly not the only solution to design a robo-pigeon for introducing controlled variables into a flock. There are several potential different ways to design according to animals nature, among which magnetoreception (Mora et al., 2004) in homing pigeons is the most representative.

Birds evolved primarily in three-dimensional environments unlimited by terrestrial or marine impediment and in many species driven by season, and their ability of direction-finding has remained a wonder. Avian use of the geomagnetic field was proposed in the mid-nineteenth century and first demonstrated in 1966 for European robins (Wiltschko and Merkel, 1966). The geomagnetic field can be used as a compass for direction, and/or for navigation as one of the elements in a mental "map" which aids in setting a course (Bookman, 1977). Though mature birds had both the innate geomagnetic compass and the "map", some juvenile birds without route experience or memory were found not to have a "map" but only their innate geomagnetic compass (Munro et al., 1997). This implied if the innate geomagnetic compass could be manipulated artificially, it might be possible to modulate a bird's flight behavior.

Many species of birds have magnetite particles concentrated in the ethmoid region that is at the root of the nose. These particles respond to a high-intensity magnetic pulse, changing orientation (Davila et al., 2003; Fleissner et al., 2003). Researchers had tried to modulate flight trajectories by altering the magnetic field surrounding the birds, thus the magnetite particles. The studies with homing pigeons equipped with magnets glued to their backs (Keeton, 1971) or a pair of small coils around their heads (Walcott and Green, 1974) showed that the reversed polarity of magnets or coils could cause disorientation when the birds were released especially under overcast. However, magnetite data have recently been questioned due to possible laboratory contamination (Edelman et al., 2015). A very different, chemically-based source of directive magnetism has been mooted. The argument indicates this field is worthy of further studies and tests that will understand the biological mechanism and an inspiration for the robo-pigeon design.

\subsection{Potential applications of robo-pigeons}

For engineering, the mechanism behind the instinct of bird flocks is quite informative for algorithm designing in drone swarms (Caggiano et al., 2018). For example, comparing the human-made aerial system to the natural behavior of bird flocks, the phenomenon of seeing neighbors in similar movement could be further developed in the field of aerial swarm robotics. One of the most appealing characteristics of collective behaviors in bird flocks is that decisions are made based on local information such as visual perception. In contrast, in the human-made aerial system, the decisions rely on centralized control. The latter bears a significant risk because agents lack the autonomy to make their own decisions in failure cases, such as communication outage. That raises the question: how an individual makes its own decision when it is involved in a flock? The approach to study animals' collective behavior using robo-pigeon could help to find the answer.

In robo-pigeon, the locomotion-related functional area in the midbrain has been allocated (Cai et al., 2015), and appropriate stimulation methods have been elaborated (Schneider and Necker, 1996). Based on these achievements, this work was the first to introduce manual controlled variables into a flying flock under natural conditions. Though only variables related to direction change were investigated, there are much broader possibilities. Recent studies revealed some midbrain circuits that set locomotor speed and gait selection in mammals (Caggiano et al., 2018) and the circuits in the caudal brainstem related to locomotor speed control (Capelli et al., 2017), which suggested that animals locomotion could be faster by direct stimulation over the brain regions with motor speed regulation function, or by increasing the stimulation frequency after inducing action onset. These recent findings may inspire designing controlled variables related to flight speed, which confidently makes robo-pigeons more helpful in collective behavior of bird flocks.

\section{Author contributions}

H.W., L.C., and Z.D. conceived and designed the experiments; H.W., L.C., and J.W. performed the experiments; H.W. and K.F. analyzed the data; J.W. and L.W. contributed reagents and materials; H.W. and L.C. wrote the paper. The founding sponsors had no role in the study; in the collection, analyses, or interpretation of data; in the writing of the manuscript, and in the decision to publish the results.

\section{Ethics approval and consent to participate}

All experimental animals operating procedures are in line with the Guide of Laboratory Animal Management Ordinance of China and are approved by the Jiangsu Association for Laboratory Animal Science (Jiangsu, P. R. China).

\section{Acknowledgments}

This work was funded by the National Natural Science Foundation of China (61973159, 61375096, 31500858) and the foundation of Jiangsu Provincial Key Laboratory of Bionic Functional Materials (NJ2019015).

\section{Conflict of Interest}

The authors declare no conflict of interest.

\section{Supplementary material}

Supplementary material associated with this article can be found, in the online version, at https://jin.imrpress.com/EN/ 10.31083/j.jin.2020.03.159. 
Submitted: May 21, 2020

Revised: September 14, 2020

Accepted: September 14, 2020

Published: September 30, 2020

\section{References}

Aravanis, A. M., Wang, L. -P., Zhang, F., Meltzer, L. A., Mogri, M. Z., Schneider, M. B. and Deisseroth, K. (2007) An optical neural interface: In vivo control of rodent motor cortex with integrated fiberoptic and optogenetic technology. Journal of Neural Engineering 4, S143.

Attanasi, A., Cavagna, A., Del Castello, L., Giardina, I., Grigera, T. S., Jelić, A., Melillo, S., Parisi, L., Pohl, O. and Shen, E. (2014) Information transfer and behavioural inertia in starling flocks. Nature Physics 10, 691-696.

Bajec, I. L. and Heppner, F. H. (2009) Organized flight in birds. Animal Behaviour 78, 777-789.

Bookman, M. A. (1977) Sensitivity of the homing pigeon to an Earthstrength magnetic field. Nature 267, 340-342.

Caggiano, V., Leiras, R., Goñi-Erro, H., Masini, D., Bellardita, C., Bouvier, J., Caldeira, V., Fisone, G. and Kiehn, O. (2018) Midbrain circuits that set locomotor speed and gait selection. Nature 553, 455-460.

Cai, L., Dai, Z., Wang, W., Wang, H. and Tang, Y. (2015) Modulating motor behaviors by electrical stimulation of specific nuclei in pigeons. Journal of Bionic Engineering 12, 555-564.

Capelli, P., Pivetta, C., Esposito, M. S. and Arber, S. (2017) Locomotor speed control circuits in the caudal brainstem. Nature 551, 373-377.

Chen, D., Liu, X., Xu, B. and Zhang, H. -T. (2017) Intermittence and connectivity of interactions in pigeon flock flights. Scientific Reports 7 , $1-11$.

Codling, E. A., Pitchford, J. W. and Simpson, S. D. (2007) Group navigation and the "many-wrongs principle" in models of animal movement. Ecology 88, 1864-1870.

Davila, A. F., Fleissner, G., Winklhofer, M. and Petersen, N. (2003) A new model for a magnetoreceptor in homing pigeons based on interacting clusters of superparamagnetic magnetite. Physics and Chemistry of the Earth, Parts $a A / B / C$ 28, 647-652.

Dimock, G. and Selig, M. (2003) 'The aerodynamic benefits of selforganization in bird flocks,' . 41st Aerospace Sciences Meeting and Exhibit. Reno, Nevada.

Edelman, N. B., Fritz, T., Nimpf, S., Pichler, P., Lauwers, M., Hickman, R. W., Papadaki-Anastasopoulou, A., Ushakova, L., Heuser, T., Resch, G. P., Saunders, M., Shaw, J. A., Keays, D. Aet al. (2015). No evidence for intracellular magnetite in putative vertebrate magnetoreceptors identified by magnetic screening. Proceedings of the National Academy of the United States of America 112, 262-267.

Erickson, J. C., Herrera, M., Bustamante, M., Shingiro, A. and Bowen, T. (2015) Effective stimulus parameters for directed locomotion in Madagascar hissing cockroach biobot. PLoS ONE 10, e0134348.

Fleissner, G., Holtkamp-Rötzler, E., Hanzlik, M., Winklhofer, M., Fleissner, G., Petersen, N. and Wiltschko, W. (2003). Ultrastructural analysis of a putative magnetoreceptor in the beak of homing pigeons. Journal of Comparative Neurology 458, 350 -360.

Fry, S. N., Rohrseitz, N., Straw, A. D. and Dickinson, M. H. (2008) TrackFly: virtual Virtual reality for a behavioral system analysis in freeflying fruit flies. Journal of Neuroscience Methods 171, 110-117.

Heppner, F. H. (1974) Avian flight formations. Bird-Banding 45, 160-169.

Keeton, W. T. (1971). Magnets interfere with pigeon homing. Proceedings of the National Academy of Sciences of the United States of America 68, 102-106.

Kingsbury, M. A., Kelly, A. M., Schrock, S. E. and Goodson, J. L. (2011) Mammal-like organization of the avian midbrain central gray and a reappraisal of the intercollicular nucleus. PLOS ONE 6, e20720.

Kobayashi, N., Yoshida, M., Matsumoto, N. and Uematsu, K. (2009) Artificial control of swimming in goldfish by brain stimulation: Confirmation of the midbrain nuclei as the swimming center. Journal of Neuroscience Letters $\mathbf{4 5 2}$, 42-46.
Li, L., Huang, L. and Wu, J. (2016) Flocking and invariance of velocity angles. Mathematical Biosciences and Engineering 13, 369.

May, R. M. (1979) Flight formations in geese and other birds. Nature 282, 778-780.

Medina, L. and Reiner, A. (1997) The efferent projections of the dorsal and ventral pallidal parts of the pigeon basal ganglia, studied with biotinylated dextran amine. Neuroscience 81, 773-802.

Mora, C. V., Davison, M., Martin Wild, J. and Walker, M. M. (2004) Magnetoreception and its trigeminal mediation in the homing pigeon. $\mathrm{Na}$ ture 432, 508-511.

Munro, U., Munro, J. A., Phillips, J. B., Wiltschko, R. and Wiltschko, W. (1997). Evidence for a magnetite-based navigational 'map' in birds. Naturwissenschaften 84, 26-28.

Nagy, M., Akos, Z., Biro, D. and Vicsek, T. (2010) Hierarchical group dynamics in pigeon flocks. Nature 464, 890.

Nathan, A. and Barbosa, V. C. (2008) V-like formations in flocks of artificial birds. Artificial Life 14, 179-188.

Sato, H. and Maharbiz, M. M. (2010) Recent developments in the remote radio control of insect flight. Frontiers in Neuroscience 4, 199.

Schneider, A. and Necker, R. (1996) Electrophysiological investigations of the somatosensory thalamus of the pigeon. Experimental Brain Re search 109, 377-383.

Schultheiss, P., Buatois, A., Avarguès-Weber, A. and Giurfa, M. (2017) Using virtual reality to study visual performances of honeybees. Current Opinion in Insect Science 24, 43-50.

Seo, J., Choi, G. J., Park, S., Lee, J., Baek, C., Jang, J., Lim, J., Shin, S., Seo, K. and Seo, J. -M. (2017). Wireless navigation of pigeons using polymer-based fully implantable stimulator: A pilot study using depth electrodes. IEEE 2017, 917-920.

Stowers, J. R., Hofbauer, M., Bastien, R., Griessner, J., Higgins, P., Farooqui, S., Fischer, R. M., Nowikovsky, K., Haubensak, W. and Couzin, I. D. (2017) Virtual reality for freely moving animals. Nature Methods 14, 995.

Su, X., Huai, R., Yang, J., Wang, H. and Lv, C. (2012) Brain mechanism and methods for robo-animal motor behavior control. Scientia Sinica Informationis 42, 1130-1146.

Talwar, S. K., Xu, S., Hawley, E. S., Weiss, S. A., Moxon, K. A. and Chapin, J. K. (2002) Rat navigation guided by remote control. Nature 417, 37-38.

Vicsek, T. and Zafeiris, A. (2012) Collective motion. Physics Reports 517, 71-140.

Walcott, C. and Green, R. P. (1974). Orientation of homing pigeons is altered by a change in the direction of an applied magnetic field. Science 184, 180-182.

Wang, H., Li, J., Cai, L., Wang, C. and Shi, A. (2018) Flight control of robo-pigeon using a neural stimulation algorithm. Journal of Integrative Neuroscience 17, 337-342.

Weimerskirch, H., Martin, J., Clerquin, Y., Alexandre, P. and Jiraskova, S (2001) Energy saving in flight formation. Nature 413, 697-698.

Weitz, S., Blanco, S., Fournier, R., Gautrais, J., Jost, C. and Theraulaz, G. (2012) Modeling collective animal behavior with a cognitive perspective: A methodological framework. PLoS ONE 7, e38588.

Wilson, D. S. and Wilson, E. O. (2007) Rethinking the theoretical foundation of sociobiology. The Quarterly Review of Biology 82, 327-348.

Wiltschko, W. and Merkel, F. W. (1966). Orientierung zugunruhiger Rotkehlchen im statischen Magnetfeld. Verhandlungen der deutschen Zoologischen Gesellschaft. 59, 362 -367. (In German)

Yang, J., Huai, R., Wang, H., Li, W., Wang, Z., Sui, M. and Su, X. (2017) Global positioning system-based stimulation for robo-pigeons in open space. Frontiers in Neurorobotics 11, 40.

Zhang, H. -T., Chen, Z., Vicsek, T., Feng, G., Sun, L., Su, R. and Zhou, T. (2014) Route-dependent switch between hierarchical and egalitarian strategies in pigeon flocks. Scientific Reports 4, 5805.

Zhao, K., Wan, H., Shang, Z., Liu, X. and Liu, L. (2019) Intracortical microstimulation parameters modulate flight behavior in pigeon. Journal of Integrative Neuroscience 18, 23-32. 